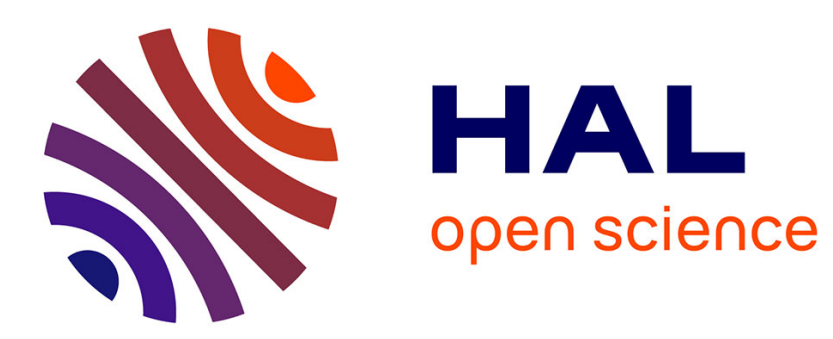

\title{
Modelling by percolation theory of the behaviour of natural coral used as bone substitute
}

\author{
Yves Barbotteau, J.L. Irigaray, J.J. Mathiot
}

\section{To cite this version:}

Yves Barbotteau, J.L. Irigaray, J.J. Mathiot. Modelling by percolation theory of the behaviour of natural coral used as bone substitute. Physics in Medicine and Biology, 2003, 48, pp.3611-3623. in2p3-00021553

HAL Id: in2p3-00021553

https://hal.in2p3.fr/in2p3-00021553

Submitted on 21 Apr 2004

HAL is a multi-disciplinary open access archive for the deposit and dissemination of scientific research documents, whether they are published or not. The documents may come from teaching and research institutions in France or abroad, or from public or private research centers.
L'archive ouverte pluridisciplinaire HAL, est destinée au dépôt et à la diffusion de documents scientifiques de niveau recherche, publiés ou non, émanant des établissements d'enseignement et de recherche français ou étrangers, des laboratoires publics ou privés. 


\title{
Modelling by percolation theory of the behaviour of natural coral used as bone substitute.
}

\author{
Y. Barbotteau* ${ }^{*}$, J.L. Irigaray** and J.F. Mathiot** \\ *Department of Quantum Science and Energy Engineering \\ Graduate School of Engineering \\ Tohoku University \\ Aoba-ku, Aramaki, Aza-Aoba 01, Sendai 980-8579, Japan \\ ** Laboratoire de Physique Corpusculaire \\ Universite Blaise Pascal - IN2P3/CNRS \\ Avenue des Landais, 63177 Aubiere cedex, France
}

\begin{abstract}
.
We investigate the relevance of site-bond percolation theory to model the resorption and ossification of natural coral implanted in bones. The first step of the process is the vascularisation of the implant seen here as a percolation threshold. The resorption and ossification can thus take place by activation of unoccupied sites. We compare our results with previously obtained experimental data on implants in cortical bones of porcine. Very encouraging results are obtained.
\end{abstract}

Submitted to: Phys. Med. Biol.

PCCF RI 03-07

$\S$ To whom correspondence should be addressed (yves.barbotteau@qse.tohoku.ac.jp) 


\section{Introduction}

The ability of human body to regenerate bony tissues that are lost or damaged is limited. In the case of important bony defects, autogenous bone graft is considered as suitable transplant material because differences in biocompatibility and the risk of transferring virus from one individual to another are non-existent. Removal of the bone graft creates additional surgical trauma. Allogenic and xenogenic bone grafts represent alternatives but several problems are generally associated with them such as in vivo resorption, virus transfer, considerable care, high cost and regular immuno-defensive reaction. For all these reasons, bone substitutes arise a growing interest and are frequently used in orthopaedic surgery. They are alternative to autogenic, allogenic and xenogenic bone grafts. One hopes that they are replaced gradually and completely by neoformed bone with the same bone characteristics at the end of the restoration process.

Natural coral, submitted to rigorous protocols of preparation and purification, can be used as a replacement biomaterial for bone grafts both in orthopaedic surgery and maxillo-cranio-facial surgery. It can replace bony tissue without inappropriate response for the human body (biocompatibility) ; it develops a chemical bond to the bone surface (bioactivity) and is able to form bony tissue when it is in contact with bone (osteoconductivity) [1]. Histological studies show that pieces of coral implanted in osseous sites are progressively replaced by neoformed bone tissue [1]. Some corals present strong architectural similitude with osseous tissue. The most promising is Porites which forms colonies and is common throughout the Caribbean area, including Bahamas, Bermuda and Brazil.

We shall investigate in this study the relevance of percolation theory to simulate coral resorption and its replacement by bone. Our motivation is to identify the very few relevant parameters which govern these processes without entering into the details of the chemical or biological microscopic processes. Of course, the interpretation of these parameters, together with their precise value, should thus be related in a second step to biological, chemical or physical processes.

\section{General presentation}

\subsection{Present knowledge}

According to the clinical application, a fast or slow resorption of the implant is required. For example, if the biomaterial is used for alveolar ridge increasing, degradation is not desired. In most cases, fast resorption is however preferable. For a given material, the porosity is an essential parameter because a macro-porous biomaterial can be colonized by bony cells and used as tissue expensor.

Coral and nacre are both natural calcium carbonate (aragonite) :

- Nacre is able to form a tight bond to bone without soft and fibrous tissue formation [2]. It is gradually and centripetally dissolved but not resorbed by cells because it is not porous [3]. 
- Coral has architectural properties, such as interconnected macro-porosity, adapted to biological requirements of the receiver bone. Natural coral implanted into bony tissue is gradually resorbed and replaced by newly formed bone.

Guillemin et al [4] have compared the fate of two Madreporian corals, Porites and Acropora. These materials are identical in composition $\left(\mathrm{CaCO}_{3}\right)$ but differ in porosity (50\% and $10 \%$, respectively). Quantitative results show that larger the porosity volume, greater is the coral resorption as well as the new bone apposition.

Patat and Guillemin [5] have studied Porites coral. They show that histologically, the bony neoformation processes are carried out in five steps which follow one and another and are overlapped as the resorption front and calcification are progressing. The first step corresponds to invasion of biomaterial by blood cellular elements or osseous cells. The second step is the establishment of a vascularisation. The third step is characterized by the biomaterials resorption by osteoclasts. The fourth concomitant step corresponds to osteoblastic affixing responsible of osseous neoformation. The last step is the neoformed bone remodelling.

Very little information exists on the coral resorption time. It seems to depend on the animal species which are used. When the implant is placed in the cortex of pig femur, $64 \%$ of the coral implant is resorbed after 1 month, whereas in sheep the percentage is $93 \%$ [4]. Roux and coworkers [6] report almost complete resorption after one year in $50 \%$ of cases when coral was used to fill craniotomy holes in humans. Larger implants used in humans are still visible by X-ray positive after 4 years [7].

\subsection{Experimental data}

We shall point out here some experimental results which will be relevant for our theoretical simulation. We notice that most of the results presented here are obtained by nuclear physics methods. They offer, in biomaterials field, many advantages [8]. These data are very important since they allow the calibration of theoretical modelling.

The coral we studied is a Porites Lutea from the Caribbean seas. Its geometrical characteristics are : cylinder $6 \mathrm{~mm}$ in diameter and $14 \mathrm{~mm}$ in length with a porosity around $50 \%$, a mean pore size about $150 \mu \mathrm{m}$ and interconnecting pores. Its architecture (shape and size) presents large similarities with bony tissue and thus justifies its use in osseous surgery : orthopaedic, dental, ...

Several studies of in vivo coral implant behaviour have been carried out by our team. We use radioactive tracers [9] to study the kinetic of implant resorption and its ossification. Both NAA (Neutron Activation Analysis) and PIXE (Particle Induced X-ray Emission) analysis methods allow to know the elementary composition of implant versus time of implantation [10]. These methods are very sensitive and enable to quantify the trace elements which have an important role in biology (such as iron to highlight the vascularisation process [11]) and to observe bone/materials interface. Moreover, we have observed the evolution of implant crystalline structure by X-ray diffraction [12]. 
The results we obtained suggest that resorption and ossification processes can be decomposed into two successive phases:

(i) a latent phase where the mineral composition and crystalline structure of biomaterial are preserved. This phase corresponds to the invasion of blood cells and to the establishment of implant vascularisation.

(ii) a phase where biomaterial resorption and bone remodelling are simultaneous. These two remodelling seem to be correlated.

We reproduce in table 1 results obtained after implantation of coral in cortical bone of porcine. The amount of coral remaining, $\left(\sigma_{\text {coral }}(t)\right)$, is calculated thanks to a radioactive tracer : ${ }^{85} \mathrm{Sr}^{*}$ while bone formation, $\left(\sigma_{\text {bone }}(t)\right)$, is calculated starting from the phosphorus concentration.

Table 1. Percentage of coral remaining $\left(\sigma_{\text {coral }}(t)\right)$ and bone formation $\left(\sigma_{\text {bone }}(t)\right)$ versus implantation time (in weeks).

\begin{tabular}{lccccccc}
\hline weeks & 1 & 2 & 3 & 4 & 6 & 10 & 24 \\
\hline$\sigma_{\text {coral }}(t)$ & $99 \pm 4$ & $79 \pm 12$ & $48 \pm 10$ & $28 \pm 6$ & $12 \pm 2$ & $2 \pm 2$ & 0 \\
$\sigma_{\text {bone }}(t)$ & 0 & $11 \pm 7$ & $44 \pm 20$ & $66 \pm 16$ & $81 \pm 7$ & $91 \pm 5$ & $97 \pm 7$ \\
\hline
\end{tabular}

\section{Percolation theory}

\subsection{Main features}

Percolation theory was introduced by Broadbent and Hammersley in 1957 [13]. They proposed to solve the following problem : What is the probability that the centre of a porous stone immersed in a fluid gets wet? This refers to the propagation of a fluid in a medium formed by stone sites. Some sites are connected by channels or bonds, and may be opened or closed in a random way. The recent development of percolation theory is related to the constant improvement of computers. It is applied to fields as different as epidemiology, economy or nuclear physics.

We shall use in this study percolation theory to simulate the biomaterial resorption. The vascularisation phase of these implants presents indeed strong analogies with this initial problem.

The percolation is a problem of communication in a wide medium where a large number of sites are distributed randomly to relay information locally. These sites communicate between them by bonds whose effectiveness is also randomly chosen. According to the proportion of active connections is (or not) higher than a threshold value, there is (or not) a possibility of transmitting information at long distances. Below the percolation threshold, information remains confined in a small area where it has appeared. When the percolation threshold is reached, information percolates and is 
spread to the whole system. The information term is taken here in its broadest sense; it can represent a physical or biological property as well as a fluid.

In our study, we shall use in the following a site-bond percolation model. The occupied sites will be associated with the pores present in the coral, while the activated bonds will represent the vascularisation of these sites. We shall be interested by the different situations that can occur depending on the values of a few, macroscopic parameter which will govern the formation, or absence, of a percolation cluster.

\subsection{Usual definitions}

Let us first describe a simple model of percolation in order to present the most important definitions. The readers can consult, for instance, the articles of Welsh [14] or Essam [15] to obtain more information on percolation theory.

Let $N$ be a system of $L \in \mathbf{R}^{d}$ in extension, described with a $\Gamma \ll L$ resolution fixed by observer and chosen according to the system under consideration. This scale should be related to biological, chemical or physical considerations.

The discretisation is carried out by a paving in identical cells of volume $a^{d}$. The state of a site is chosen randomly, and the sites are statistically independent: each of them is occupied with the same probability $p$, therefore empty with the probability $(1-p)$. The probability $p$ that a site is occupied coincides with the site concentration if the network is sufficiently large. Two neighboring occupied sites can be linked by a bond with a conditional probability $q$. Each bond is statistically independent and identical (with a length $a$ ). If $p=1, q=1$ or $p$ and $q$ are different from 1 , this model is respectively called bond, site or site-bond percolation.

A cluster is an assembly of occupied sites, linked bonds or occupied sites linked by bonds respectively for site, bond or site-bond percolation. In the site percolation model, the sites of a cluster will have to be surrounded by a succession of neighboring occupied sites; in the case of the model of bond percolation, two bonds of a cluster will have to be linked by a chain of bonds. In the site-bond percolation model, a bond can only exist between two occupied sites. In an infinite network, one calls percolation threshold the concentration $p_{c}$ for which the first infinite cluster appears. This concentration has an universal value, depending only on the chosen percolation model, the geometry and the dimension $d$ of the network but not on the biological, chemical or physical interpretation of the occupied sites or bonds. In a finite network of characterics length $L$, one introduces the concept of percolating cluster, whose definition depends on the physical context, the model and the geometry of the network: we can define it for instance by the existence of a cluster connecting various edges of the network.

\section{Method}

We have carried out two kinds of modelling: a first one in two dimensions (2D) and a second one in three dimensions (3D). Obviously, 2D simulations take a processing 
time much shorter than 3D simulations. This time saving enabled us to validate some assumptions and to check that percolation theory is indeed adequate to simulate the behaviour of coral implant.

In our first simulations, we investigate the $2 \mathrm{D}$ model for which it is easy to have a visual representation. The implants will be schematically represented by a rectangle (see figure 1).

The resolution $\Gamma$ is fixed to $150 \times 150 \mu \mathrm{m}^{2}$. By definition, it is the area of network site. An occupied site represents a pore which can be colonized by bone cells; an empty site represents coral or bone. This resolution takes into account several parameters like for instance the size of the cells or the porosity of coral. The larger cells are osteoclasts with a size range between 10 to $100 \mu \mathrm{m}$ in diameter. They secrete carbonic anhydrase which is an enzyme responsible of coral resorption. The coral we used in this study has pores of about $150 \mu \mathrm{m}$ in diameter. Since this resolution is significantly larger than the cellular scale, it is expected that the main characteristics of the vascularisation process will be determined by global parameters. This is precisely the aim of the percolation model.

The modelling of coral resorption and ossification is carried out in two phases:

- the vascularisation of implant;

- the resorption and bone remodelling.

\subsection{Implant vascularisation}

As we already mentioned, biomaterial will be represented by empty sites and its pores by occupied sites. Let $p$ be the ratio of occupied sites. Here two states (occupied or non-occupied) are supposed to correspond to identical occupancy in real space so that the probability $p$ is identical to the porosity of the biomaterial. When two occupied sites (pores) are linked by a bond, we consider that they are vascularised. We define a cluster of activated bonds like an ensemble of vascularised pores. We shall denote by $q$ the probability to activate a bond between two occupied sites.

From a pratical point of view, a vascularised pore is randomly chosen among all vascularised pores. The activation of a link starting from this pore is chosen with the probability $q$. The number of iteration necessary to simulate one week of implantation is noted $n_{0}$.

In 2D simulations, vascularisation starts on two opposite edges of a rectangle (usually the lengths) and extends, at each iteration, inside the network. When these two vascularisation sources meet, the ensemble of activated bonds is the percolating cluster.

The figures 1 (left column) show the vascularisation steps. Empty sites (biomaterial) are black, occupied sites (pores) are white and activated bonds are red (vascularised pores). The first figure is the initial implant filled randomly with $p=0.5$ (porosity of $50 \%$ ). Other figures simulate the vascularisation process. On the last figure of the left column, we are about to obtain the percolating cluster. 

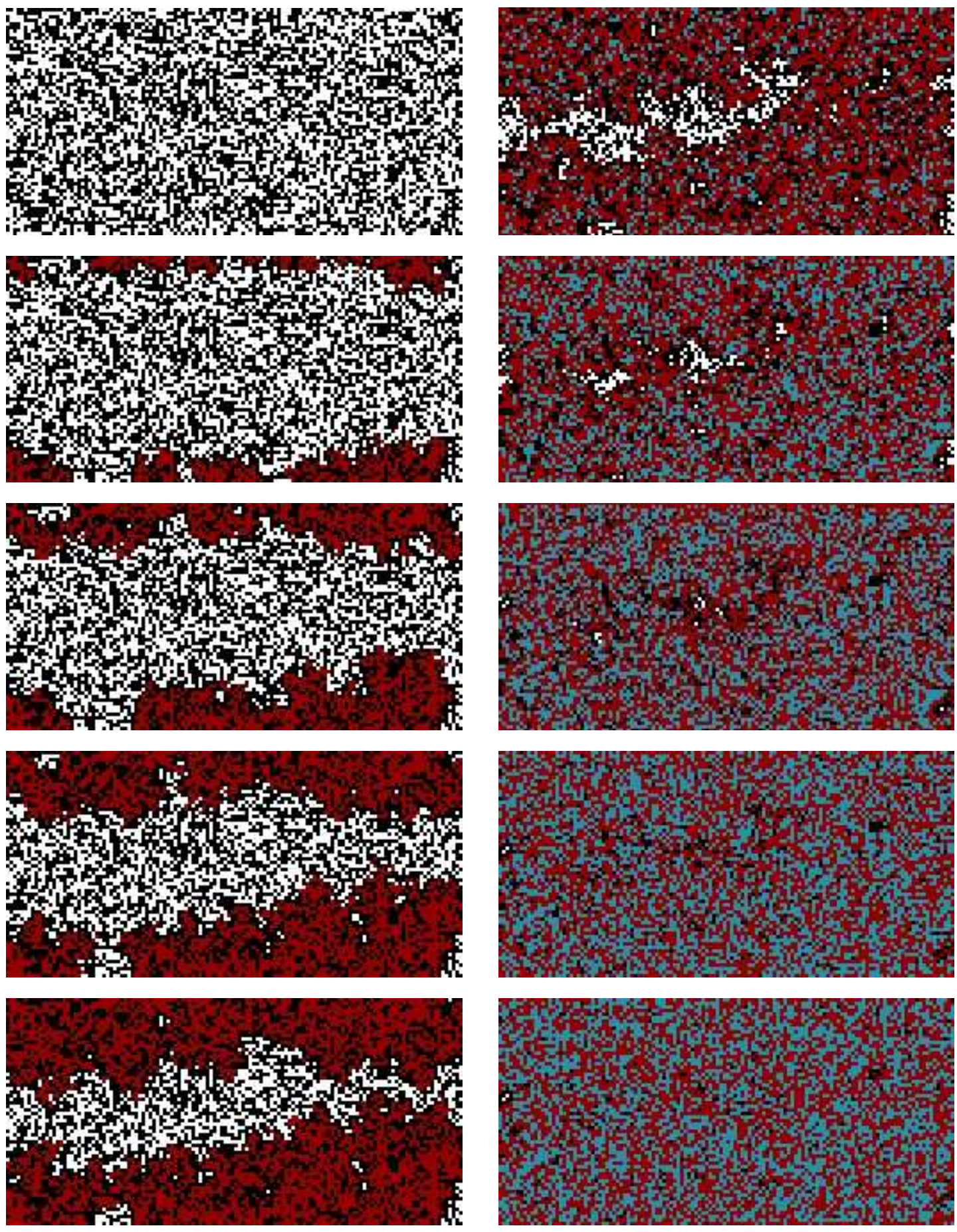

Figure 1. 2D simulation of the vascularisation, resorption and bone remodelling in an implant with a porosity of $50 \%$.

\subsection{Resorption and bone remodelling}

Once the percolating cluster is established, simulation of implant resorption and ossification starts, vascularisation continues simultaneously. Both mechanisms of 
ossification and resorption are controlled by the same parameter, denoted by $r$. It is the probability that a vascularised pore (containing osseous cells) becomes a bone site (ossification) or fills an empty site among its close neighbours (resorption of biomaterial or bone). The probabilities of ossification or bone resorption are the same in order to obtain a final bone porosity of $50 \%$, whatever the initial implant porosity is. We can thus imitate in this way the process of bone remodelling. Figure 1 (right column) shows the resorption/ossification steps. Empty sites representing bone are blue.

\section{Results and discussion}

For our 3D simulations, the rules are almost the same as in 2D. The implant representation and the percolation criterion are only different. Implants are represented by right-angled parallelepipeds with a square base. In order to be more realistic, the vascularisation step starts simultaneously on the six faces of the parallelepiped. The percolating cluster is said to be reached when $50 \%$ of empty sites are bonded. The implant is thus considered as vascularised. We shall discuss thereafter the influence of this criterion.

\subsection{Comparison with experimental results}

Implants are viewed like a $40 \times 40 \times 100$ network. In figure 2 , we compare experimental results with the results of our simulations for the resorption and ossification in coral cylinders implanted in cortical bone of porcine. Dots are experimental results already given in table 1 and the full lines are obtained by averaging 90 simulations.
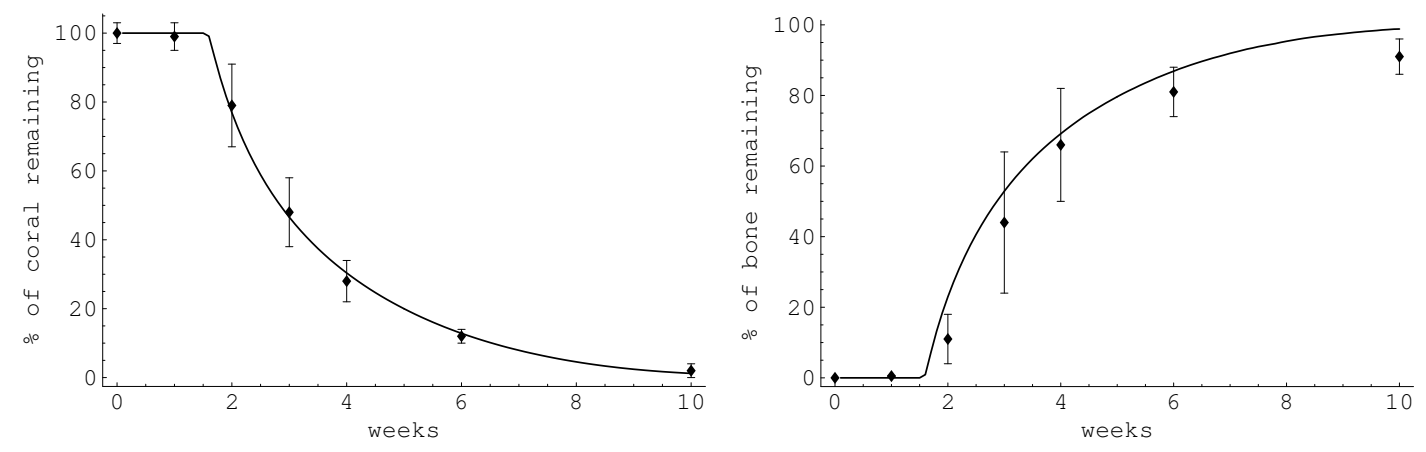

Figure 2. Comparison with experimental results: resorption of coral implant (left) and ossification (right). Dots are experimental results and full lines are simulation results.

As we already mentioned, the parameter $p$ can be identified, in our model, with the porosity of the biomaterial. The parameter $q$ represents the probability to activate a bond between two occupied sites. In the vascularisation process, it corresponds to the 
probability to vascularise a neighboring site, from a site already vascularised. We chose the parameter $q$ to be independent of the implantation site. Its value is related only to biomaterial physico-chemical properties. For instance, the nature of biomaterial (coral, hydroxyapatite, ...), and the biomaterial porosity (pore size, interconnected pore or not, ...) seem to be relevant properties. The parameter $r$ depends on the nature of biomaterial. For instance, the resorption of biomaterial may depend of the carbonic anhydrase ability; this enzyme is secreted by osteoclasts to dissolve the biomaterial. Then, $p, q$ and $r$ parameters are only dependent on the biological, chemical or physical properties of the implant, but not on its size or geometry. The $p$ and $q$ parameters should also depend on animal characteristics such as species, race and possibly sexe and age.

The last parameter is the time scale, noted $n_{0}$. It fixes the number of iteration necessary to simulate one week of implantation. It is inherent to any numerical simulation in which the time scale should be fixed by comparison of the numerical simulation with experimental data. Since we do not relate in this first study the parameter $q$ to properties of the biomaterial, we can take $q$ equal to one, in order to speed up the computation time. This is possible since, as we mentioned above, the overall time scale cannot be fixed by the simulation but shoud be determined by a direct comparison with experimental data. The parameter $q$ is defined up to a constant scale. It is checked explicitly in our simulation since the results do not depend on $q$ and $n_{0}$ independently, but on $q \times n_{0}$.

The equivalence between the number of iteration, $n$, and the time $t$ is given by:

$$
t=\frac{n}{n_{0}}
$$

The time scale parameter $n_{0}$ is, contrary to the other parameters, independent of the biological, chemical or physical properties of the implant. It may however depend on the size and the geometry of the implant (see our discussion in sec.(5.3)).

We can extract from our simulation two characteristic times. The first one is the time needed to obtain percolating cluster. It is called percolation time and denoted by $t_{p}$. The second one, noted $\tau$ and measured starting from $t_{p}$, is the time necessary to resorb half of the implant. The parameters $t_{p}$ and $\tau$ are enough to characterise the curves obtained by our simulations. Indeed, we can identify two different parts in our results:

if $t \leq t_{p}$

$$
\begin{aligned}
& \sigma_{\text {coral }}(t)=100 \\
& \sigma_{\text {bone }}(t)=0
\end{aligned}
$$

if $t>t_{p}$

$$
\sigma_{\text {coral }}(t)=100 \cdot \exp \left(-\frac{\ln 2 \times\left(t-t_{p}\right)}{\tau}\right)
$$


Modelling by percolation theory of the behaviour of natural coral used as bone substitute.10

$$
\sigma_{\text {bone }}(t)=100 \cdot\left(1-\exp \left(-\frac{\ln 2 \times\left(t-t_{p}\right)}{\tau}\right)\right)
$$

The parameters used to simulate the case of natural coral implanted in porcine bone and both characteristics times obtained are shown in table 2 .

Table 2. $p, q, r$ and $n_{0}$ parameters used for our simulations together with the characteristic times obtained.

\begin{tabular}{cccc}
\hline$p$ & $q$ & $r$ & $n_{0}$ \\
0.5 & 1 & 0.145 & $1.05 \times 10^{6}$ \\
& & & \\
$t_{p}($ weeks $)$ & \multicolumn{2}{c}{$\tau$ (weeks) } \\
$1.60 \pm 0.03$ & \multicolumn{2}{c}{$1.25 \pm 0.01$} \\
\hline
\end{tabular}

\subsection{Influence of the porosity: percolation threshold}

We have highlighted in section 2 that the resorption of natural coral implants depends on the porosity of the biomaterial. We have simulated the implant resorption with different porosities in order to determinate the percolation threshold. The network size and the parameters $q, r$ and $n_{0}$ are the same as previously, only porosity changes. For each $p$ value, we perform 90 simulations. We present in figure 3 the probability to obtain a percolating cluster in each of the simulation.

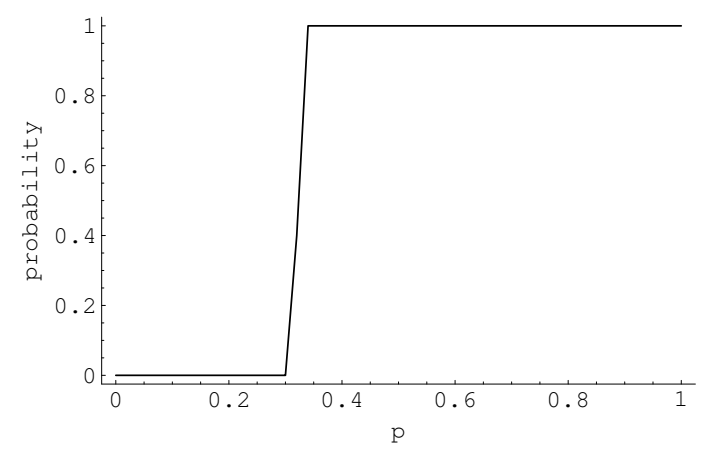

Figure 3. Probability to obtain a percolating cluster in $40 \times 40 \times 100$ network as a function of porosity.

Despite of the finite size of the network, the percolation threshold is clearly visible. Under a porosity of $30 \%$, there is no vascularisation and hence no resorption either. Above $34 \%$, there are vascularisation and resorption of the implant. 
We have calculated the evolution of the percolation time as a function of porosity. The results are given in figure 4 . We have fitted our results by the function:

$$
t_{p}=a \cdot\left(p-p_{c}\right)^{-\alpha}
$$

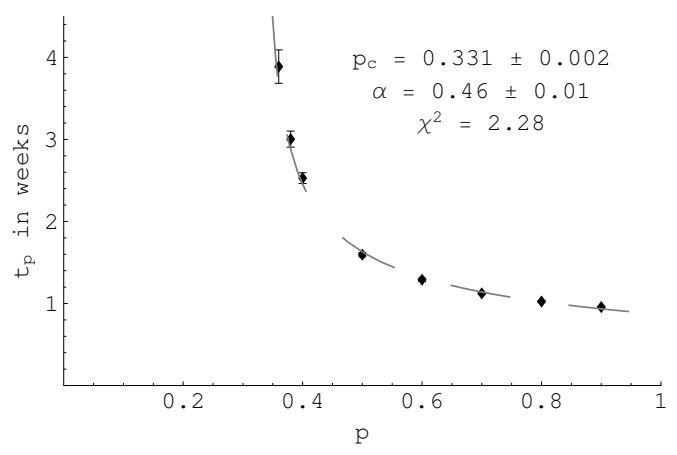

Figure 4. Percolation time $t_{p}$ evolution as a function of porosity ; the fit is represented by a dashed line.

The average value of the porosity threshold is : $p_{c}=33.1 \pm 0.2 \%$. From a theoretical point of view, this critical porosity must be independent of criterion of percolation in the case of an infinite network. In our case, some effects of finite size may appear. In order to quantify this dependency, we do again the simulation with different percolation criterions and we deduce the critical porosity for two different network sizes. The results are given in figure 5 .

(a)

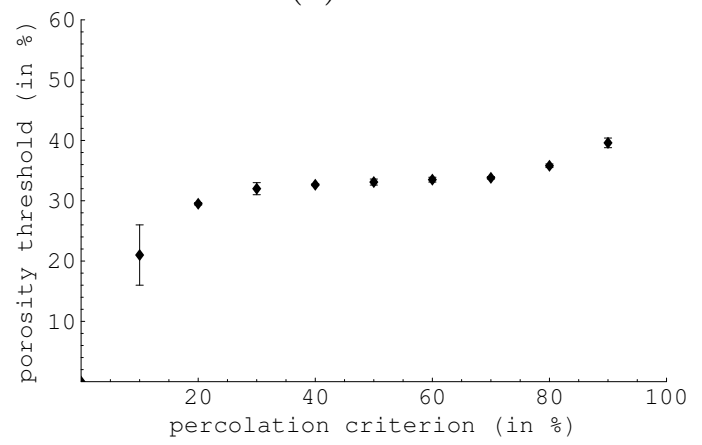

(b)

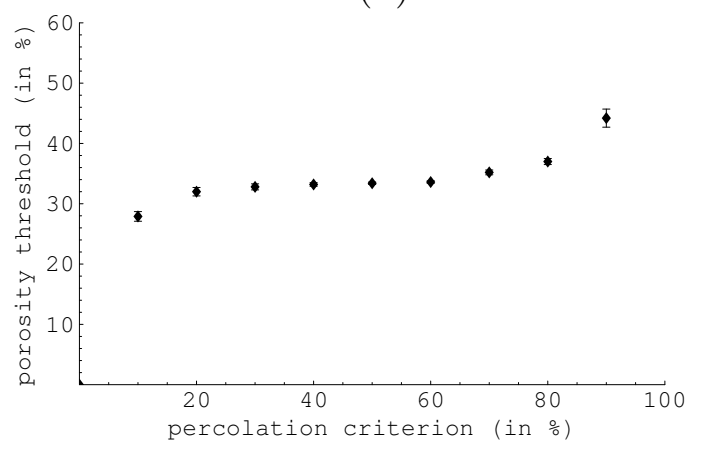

Figure 5. Porosity threshold: (a) $40 \times 40 \times 100$ network ; (b) $80 \times 80 \times 100$ network.

Despite the relatively small size of our networks, the dependence of the critical percolation is sizeable only for the extreme unrealistic values of the percolation criterions. We estimate the $p_{c}$ and $\alpha$ values by averaging the results : $p_{c}=33.1 \pm$ $0.1 \%$ and $\alpha=0.433 \pm 0.005$. 


\subsection{Implant volume and shape effects}

Experimental results show that resorption occurs well for small implant but is very slow when the volume increases. We simulate some natural coral implants with different sizes in order to study the variation of the percolation time $t_{p}$ as a function of the implant size ( $p, q, r$ and $n_{0}$ parameters are kept constant and their values are those used previously). As previously, we did 90 simulations for each tested size. The network size varies in several ways:

- length variation (a): the network length varies between 10 to 390 sites (total of 14 length variations tested) and the network base is kept constant: $40 \times 40$ sites ;

- square base variation (b): the network base varies between $10 \times 10$ to $180 \times 180$ sites, with a constant length of 100 sites (total of 7 square base variations tested);

- cubic variation (c): we have simulated a cubic network of which the size varied between $10 \times 10 \times 10$ to $180 \times 180 \times 180$ sites (total of 7 cubic variations tested).

Since we defined "our time" by the simple scaling law $n / n_{0}$, without any reference to the available phase space volume, we expect that $n_{0}$ depends on the geometry of the implant, and on its size. In our simple preliminary study, this behaviour can however be predicted in a simple way. Let $\langle R\rangle$ be an average measure of this phase space volume. Before the percolation cluster has been reached, this average phase space volume should be proportional to the number of sites, noted $n_{S}$, in the contact surface $S$ between the bone and the implant. Once the percolation threshold has been reached, the transformation of the implant should occur over the whole volume of the implants, and the average phase space volume $\langle R\rangle$ should be proportional to the number of sites, noted $n_{V}$, in the volume $V$ of the implant. We shall thus take $<R>=n_{S}$ before the percolation threshold, and $\langle R\rangle=n_{V}$ after. The ratio between phase space volume and real space volume is then, before the percolation cluster appears, equal to the $S / V$ ratio. It may be seen as a kind of efficacity of the considered process. Indeed, more this ratio will be close to one, more the time $t_{p}$ will be short.

We can thus define the renormalized times $t_{p}^{r}$ and $\tau^{r}$ as:

$$
\begin{aligned}
t_{p}^{r} & =t_{p} \frac{n_{S}^{0}}{n_{S}}, \\
\tau^{r} & =\tau \frac{n_{V}^{0}}{n_{V}},
\end{aligned}
$$

where $n_{S}^{0}$ and $n_{V}^{0}$ are the quantities corresponding to our implant of reference studied in section (5.1). The variations of the renormalized time $t_{p}^{r}$ as a function of the volume for the different variations we consider are shown on figure (6).

For an implant with fibre shape (wide length and small square base), we have found that $t_{p}^{r}$ is close to a constant for a sufficiently large length in order to avoid border effects. This is expected since in this case $t_{p}^{r}$ should be independent of the length as soon as the vascularisation process proceeds homogeneously on the whole length of the implant. This is not the case for the two other variations which are both very similar. 
For the variations of (b) and (c) types (implants which have a more massive shape), the renormalized percolation time is not anymore constant, and increases rapidly with the volume, as found experimentally.

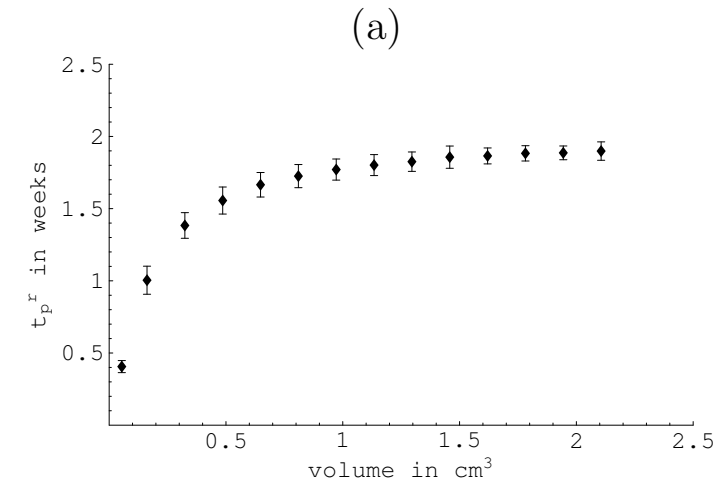

(b)

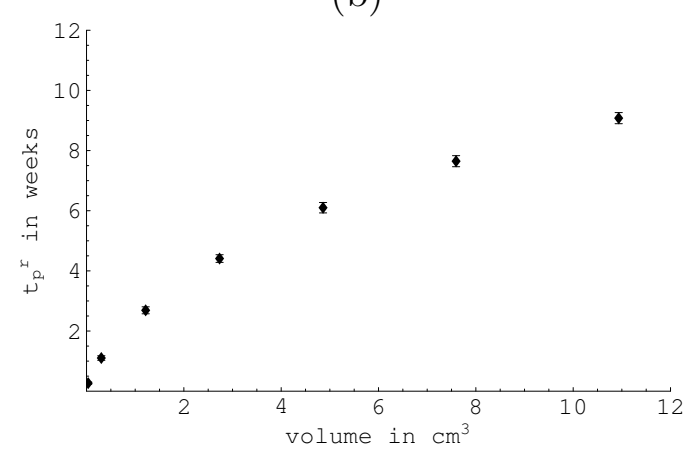

(c)

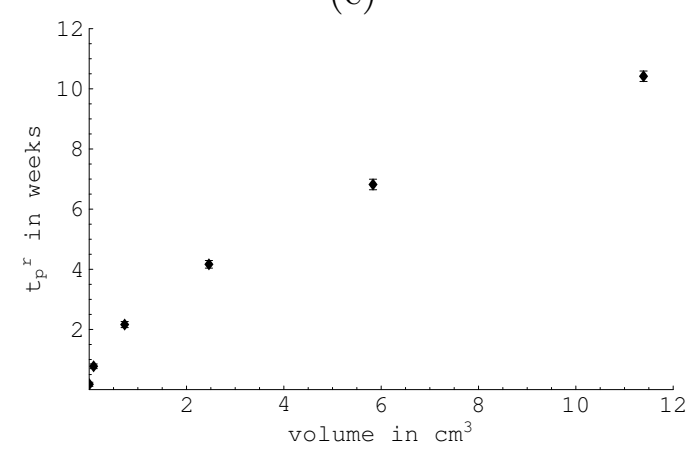

Figure 6. The renormalized characteristic times $t_{p}$, as a function of the volume in the case of (a) length, (b) square base and (c) cubic variations.

This different behavior of $t_{p}^{r}$ as a function of the volume can be related to the different behaviors of the ratio surface/volume for each variations of the size of the 
implant. We draw this ratio on figure 7 . In the case of length variation, this ratio tends quickly to its limit value. That is not the case for the (b) and (c) variation types. For this type of variation and the tested size, the $S / V$ ratios behaviours and their values are close.

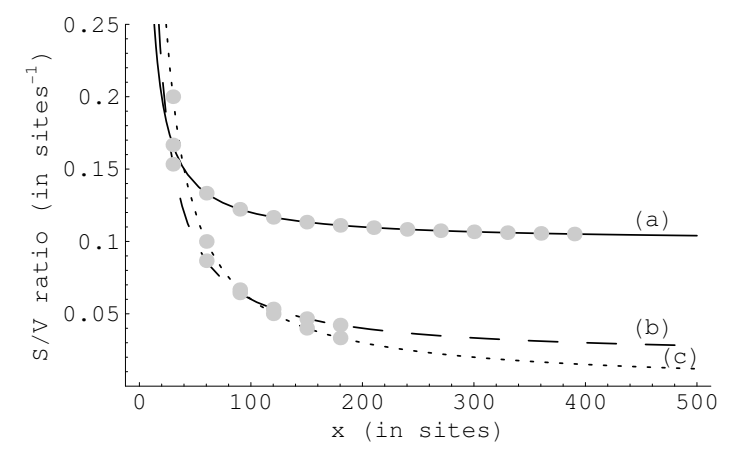

Figure 7. $S / V$ ratio values for each kind of variation of the size of the implant. The dots represent the tested size.

When the percolation cluster is reached, the evolution of the system, characterized by the renormalized half time $\tau^{r}$ is more or less homogeneous over the whole volume, so that $\tau^{r}$ should not depend on the volume of the implant. We checked that this is the case in our numerical simulation.

As for the determination of the percolating threshold, we did again all the simulations with different values of criterion. Also here, the dependency of our results with respect to the percolation criterion is weak.

\section{Conclusion}

We have proposed in this study to use percolation theory in order to simulate implant resorption and ossification. We have compared our results with experimental data for coral implants in cortical bone of porcine.

The first results are very encouraging. We have been able to model the two steps of the process. The first one is the vascularisation of the implant. Once this is done, i.e. once the percolation cluster has been reached, the implant resorption and ossification can occur simultaneously. This second step is represented as a random process on individual sites of the network.

We showed that the prediction of the vascularisation phase duration is not commonplace. However, to obtain a therapeutic success, it is necessary that vascularisation occurs fastly so that the repairing phase of bone remodelling starts as soon as possible. This kind of simulation may have a role into design of implant, with the proviso of incorporating the possibility of treating implants with an unspecified form. 
To go further, it would be of interest to test our model with hydroxyapatite implants. Indeed, hydroxyapatite is used more intensively than coral or nacre and the literature is more important. Moreover, hydroxyapatite is a synthetic material, and its properties can thus be changed at will. This is important in order to completely calibrate the parameters of our model, and to check its predictions in a wide range of situations.

The next step will be to interpret our relevant parameters in terms of biological, chemical or physical processes. Work in this direction is in progress.

\section{References}

[1] Guillemin G, Patat JL, Fourni J and Chetail M 1987 The use of coral as a bone graft substitute J. Biomed. Mater. Res. 21 557-67.

[2] Camprasse S, Camprasse G, Pouzol M, Lopez E 1990 Artificial dental root made of natural calcium carbonate (Bioracine) Clin. Mater. 5 235-50.

[3] Atlan G, Balmain N, Berland S, Vidal B and Lopez E 1997 Recontruction of human maxillary defects with nacre powder : histological evidence for bone regeneration. CR Acad Sci-Serie Iii Sci Vie 320 253-8.

[4] Guillemin G, Meunier A, Dallant P, Christel P, Pouliquen JC and Sedel L 1989 Comparison of coral resorption and bone apposition with two natural corals of different porosities J. Biomed. Mater. Res. 23 765-79.

[5] Patat JL and Guillemin G 1989 Le corail naturel utilise comme biomateriau de substitution la greffe osseuse Ann. Chir. Plast. Esthet. 34 221-5.

[6] Roux FX, Brasnu D, Loty B, George B and Guillemin G 1988 Madreporic coral : a new bone graft substitute for cranial surgery J. Neurosurg. 69 510-3.

[7] de Peretti F, Trojani C, Cambas P, Loubiere R and Argenson C 1996 Le corail comme soutien d'un enfoncement articulaire traumatique Rev. Chir. Orthop. 82 234-40.

[8] Irigaray JL, Oudadesse H and Brun V 2001 Nuclear methods to characterize biomaterials Biomaterials 22 629-40.

[9] Irigaray JL, Sauvage T, Oudadesse H, El Fadl H, Deschamps N, Lefaivre J, Barlet JP, Terver S and Tixer H 1993 Study of the mineralization of coral implanted in vivo by radioactive tracers J. Radioanal. Nucl. Chem. 174 93-102.

[10] Irigaray JL, Oudadesse H and Sauvage T 2000 Biotransformation of implanted coral in some animals studied by neutron activation analysis J. Radioanal. Nucl. Chem. 244 317-9.

[11] Braye F et al 1996 Resorption kinetics of osseous substitute : natural coral and synthetic hydroxyapatite Biomaterials 17 1345-50.

[12] Irigaray JL, Oudadesse H, Blondiaux G and Collangettes D Structure study of coral implanted in vivo by nuclear activation analysis and by X-ray diffraction,1989, Nuclear Analytical Methods in the Life Sciences International Congress of Gaithersburg, Maryland 17-21 April (USA).

[13] Broadbent SR and Hammersley JM 1957 Percolation Processes Proc. Camb. Phil. Soc. 53 629-41.

[14] Welsh DJA 1977 Percolation and related topics Sci. Prog. Oxf. 64 65-83.

[15] Essam JW 1980 Percolation theory Rep. Prog. Phys. 43 832-912. 